\title{
Kazuhiro Fujiwara, Fumiharu Kato: “Foundations of Rigid Geometry I"
}

\section{EMS, 2018, 863 pp}

\section{Torsten Wedhorn ${ }^{1}$}

Accepted: 26 April 2021 / Published online: 3 May 2021

(c) The Author(s) 2021

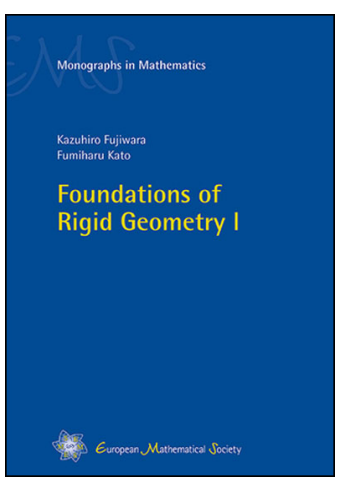

The book under review is the first volume in a book project by the authors that aims to discuss the rich overall structures of non-archimedean analytic geometry. Let me first describe what is meant by non-archimedean analytic geometry and why it is also called rigid geometry.

Hensel defined the field of $p$-adic numbers at the end of the 19th century. By now this field and, more general, non-archimedean fields have become ubiquitous not only in number theory but in many areas of mathematics. By definition, a non-archimedean field is a field $K$ endowed with a non-trivial absolute value $|\cdot|$ with values in the non-negative real numbers such that for $x, y \in K$ one has

$$
|0|=0, \quad|1|=1, \quad|x y|=|x||y|, \quad|x+y| \leq \max \{|x|,|y|\} .
$$

The last equation is stronger than the usual triangle inequality. The absolute value defines a metric on $K$ as usual by $d(x, y)=|x-y|$ and one assumes that this metric space is complete. Examples of such non-archimedean fields are $\mathbb{Q}_{p}$, the field of $p$-adic numbers, or $\mathbb{C}_{p}$, the completion of an algebraic closure of $\mathbb{Q}_{p}$.

The non-archimedean triangle inequality has some interesting consequences for the analytic, geometric, topological, and algebraic behaviour of such fields.

(1) A series $\sum_{n=0}^{\infty} a_{n}$ with $a_{n} \in K$ converges if and only if the sequence $\left(a_{n}\right)_{n}$ converges to zero.

(2) If you have a closed disc $D_{r}\left(x_{0}\right):=\left\{x \in K ;\left|x-x_{0}\right| \leq r\right\}$ of radius $r>0$ around a point $x_{0} \in K$, then every point of it is a center, i.e., for all $y_{0} \in D_{r}\left(x_{0}\right)$ one has $D_{r}\left(y_{0}\right)=D_{r}\left(x_{0}\right)$.

$\triangle$ T. Wedhorn

wedhorn@mathematik.tu-darmstadt.de

1

Darmstadt, Germany 
(3) The topological space $K$ is totally disconnected, i.e., the only connected subsets of $K$ are those that consist of a single point.

(4) The closed unit disc $D_{1}(0)$ around 0 is stable under multiplication and addition and hence defines a subring of $K$, usually denoted $O_{K}$ or $K^{\circ}$ and called the ring of integers of $K$. For instance, if $K=\mathbb{Q}_{p}$ is the field of $p$-adic integers, then $\mathbb{Q}_{p}^{\circ}=\mathbb{Z}_{p}$ is the ring of $p$-adic integers.

Already in the first half of last century the idea arose to develop a non-archimedean geometry in analogy to other geometric theory such as the theory of differentiable real manifolds or of complex analytic manifolds. But the naive approach using $p$ adic analytic functions, i.e., functions that admit locally a convergent power series expansion, encountered severe problems. As $K$ is totally disconnected, one does not have sufficient rigidity for such analytic functions. For instance, for $K=\mathbb{Q}_{p}$ the characteristic function of the unit circle $\{x \in K ;|x|=1\}$ is analytic because the unit circle is open and closed in $\mathbb{Q}_{p}$.

In the 1960's, J. Tate found a solution to this problem by defining a sufficiently large class of analytic functions and "rigidifying" the topology by defining admissibility conditions for open coverings for which global functions can be defined locally. For instance the open covering of $\mathbb{Z}_{p}$ consisting of the unit circle and its complement is not admissible. Therefore the characteristic function of the unit circle is not an admissible (in the new sense) function any more. Tate called his spaces rigid analytic spaces and this was the birth of rigid geometry.

Since then rigid geometry has undergone a huge development and has become an essential tool in number theory, algebraic geometry, and beyond. Moreover, several generalizations and variants of rigid spaces have been defined and studied such as Berkovich spaces or R. Huber's adic spaces. Examples of very active research areas, where rigid geometry and their generalizations play an important role, include arithmetic moduli spaces (e.g. Shimura varieties), the Langlands program, $p$-adic Hodge theory, or moduli of Galois representations.

For the book under review, a discovery of M. Raynaud only a few years after Tate's definition of rigid spaces is essential. Raynaud showed that the category of Tate's rigid analytic spaces (with some finiteness conditions that are not mentioned in the following) is equivalent to the category of formal models over $O_{K}$ up to certain admissible modifications.

To get a geometric idea of Raynaud's insight one has to understand that algebraic geometry views $O_{K}$ intuitively as an arbitrary small disc around a base point, i.e., an arbitrary small (complex) 1-dimensional neighborhood of a base point and that $K$ is then viewed as this small disc with the base point removed. From this point of view, a rigid space is a family of spaces over the small disc with a hole. Raynaud proved that such a rigid space can always be extended to a family of spaces over the whole arbitrary infinitesimally small disc by a so-called formal scheme and that this extension is unique up to certain well understood modifications, so called admissible blow-ups. Hence one can redefine a rigid space to be a formal scheme up to admissible blow-ups.

This is the point of view taken in this book but generalized vastly. As propagated already by Grothendieck, the book develops a relative theory of rigid spaces. As basic building blocks they use pairs $(A, I)$, where $A$ is a ring (always commutative), 
and $I \subseteq A$ is a finitely generated ideal. From the view point of Algebraic Geometry, this means that they consider a local algebraic manifold (more precisely, an affine scheme), given by the ring $A$, and a closed subspace, given by the ideal $I$, such that the open complement is quasi-compact, corresponding to the property that $I$ is finitely generated. Then $A$ has a unique topology, compatible with the ring structure, such that the powers $I^{n}$ form a basis of open neighborhoods of 0 . One also assumes that $A$ is complete as a topological abelian group. In the case $A=O_{K}$ one takes for $I$ an ideal generated by some non-zero element of absolute value $<1$. To $(A, I)$ a formal scheme is attached that one should view as a family of infinitesimal thickening of the closed subspace defined by $I$, a family whose "limit" is the algebraic manifold defined by $A$. Then locally a rigid space is defined as such a formal scheme up to certain admissible modifications of the closed subspace defined by the ideal. General rigid spaces are obtained by globalizing this construction.

The book of Fujiwara and Kato gives a thorough exposition of the fundamentals of rigid spaces. The book develops the whole theory from scratch. It is very careful in removing many unnecessary finiteness hypotheses in the existing literature that very often limited the scope of applications. For instance, the theory of formal schemes had already been developed in the 1960's by Grothendieck and his school but almost all non-trivial results were obtained under restrictive finiteness conditions that essentially excluded examples such as the ring of integers of $\mathbb{C}_{p}$. Even if one starts with objects where the classical finiteness hypotheses hold, e.g., formal schemes of finite type over $\mathbb{Z}_{p}$, fibers of morphisms between rigid spaces associated to such formal schemes yield in general rigid spaces that are not covered by the classical frame work.

The book consists of three chapters. In a Chapter 0 preliminaries are collected. It not only introduces basic language but already contains many results, especially about topological rings, that are new or difficult to find in the literature.

Chapter I is devoted to the geometry of formal schemes. It gives a very general definition of a formal scheme, the same that was already used by Grothendieck in the middle of last century. But then, instead of specializing to the noetherian case quickly, as done in the classical literature, they define various finiteness properties, that are weaker then the classical noetherianess hypothesis. Let me single out two of these properties.

The first one is that of an adic formal scheme of finite ideal type, where the basic building blocks are pairs $(A, I)$ as described above.

The second (and stronger) finiteness property for formal schemes is that of being universally adhesive, where the basic building blocks are pairs $(A, I)$ as above such that for any finitely generated $A$-module $M$ the quotient of $M$ by its $I$-torsion is of finite presentation. Moreover one demands this to remain true after passage to finitely generated $A$-algebras and completions of them. For instance, every formal scheme of finite type over the ring of integers of a non-archimedean field (or, more generally, over a valuation ring that is complete with respect to the $\varpi$-adic topology for some element $\varpi \neq 0$ of absolute value $<1$ ) is universally adhesive.

The first property yields the level of generality used to define rigid spaces in the next chapter. The second property is the framework that allows to extend, in a highly non-trivial way, several deep theorems known previously only under noetherian hypotheses. Examples of such theorems are the Theorem of formal functions and 
Grothendieck's existence theorem (called GFGA theorems in the book), or Stein factorization. Sometimes here and in the sequel the book formulates results slightly more general than in the universally adhesive case. This will be ignored in this overview.

This chapter also develops a theory of quasi-coherent modules on formal schemes and of coherent modules on universally adhesive schemes. This includes a cohomology theory for them, something that has been lacking even in the classical noetherian case.

The last chapter on rigid spaces is the main part of the volume. It introduces rigid spaces as spaces that are locally given by a qcqs adic formal scheme of finite ideal type up to admissible blow-ups. Here "qcqs" is a standard topological finiteness condition of "being not to big", a condition which is called coherent in the book. If one restricts to the class of universally adhesive formal schemes one obtains by definition the universally adhesive rigid spaces.

To say "up to admissible blow-ups" means that one considers a localization of categories. The authors show that rigid spaces, introduced by such an abstract categorical construction, can also be interpreted as a topological space together with two sheaves of rings. This is called the Zariski-Riemann triple attached to a rigid space.

Then a systematic study of rigid spaces is started. Basic properties of morphisms between rigid spaces are collected and studied. The rather subtle notion of dimensions of rigid spaces is briefly treated. The authors prove an analogue of Cartan's Theorem A and Theorem B from complex analysis for coherent sheaves on certain universally adhesive formal schemes, called Stein affinoids. The GFGA theorems of Chapter I are used to prove GAGA theorems, i.e., comparison theorems between complete families of algebraic varieties (more precisely, proper schemes) over the open complement of the closed subspace defined by the ideal $I$ and the attached rigid space.

The chapter also contains an appendix that proves that in many important cases Zariski-Riemann triples are adic spaces as defined by Huber. Moreover, another appendix shows that Berkovich spaces over non-archimedean fields can be obtained from the Zariski Riemann triples by taking Hausdorff quotients.

On the back of the book it is claimed that the book is self contained. This statement has to be qualified somewhat. The book is only self contained up to mathematical notions and techniques that are well documented in the literature. In fact, the reader is required to know quite a bit of Algebraic Geometry (such as the theory of schemes), sheaves and their cohomology, or Homological Algebra (such as derived tensor products). In addition, the book is written deliberately in an encyclopedic style with a strong emphasis on foundational definitions, techniques, and results, but with almost no examples.

This makes it an excellent and very welcome source for people already working with formal schemes and rigid spaces. But the book is maybe less suitable to work as a text book to learn rigid geometry.

A first version of the book appeared on ArXiv almost ten years ago. At that time, the title "Foundations of Rigid Geometry" was fully justified. Another foundational work in that area is A. Abbe's book on rigid spaces "Éléments de Géométrie Rigide" from 2010 which is only very briefly mentioned. Here a remark would have been welcome, that the class of "anneau idyllique", which is fundamental in Abbe's work, is a special case of the universally adhesive case mentioned above. 
In the last ten years, non-archimedean geometry has been one of the most rapidly developing areas not least because of the many new and very successful ideas introduced by Scholze together with various coauthors. This concerns new and important classes of rigid spaces such as perfectoid spaces that are not mentioned in the book, although they can be also viewed as rigid spaces in the sense of Fujiwara and Kato. Even the very fundaments of the whole area are in the process of being newly laid using recent ideas to replace topological spaces by condensed sets and completeness by solidity in the sense of Clausen and Scholze.

The book will be a very valuable source within this exciting process of advancing non-archimedean geometry. Even if the foundations of that area are currently rebuilt, the book provides a very stable high ground from which to strive further.

Funding Note Open Access funding enabled and organized by Projekt DEAL.

Publisher's Note Springer Nature remains neutral with regard to jurisdictional claims in published maps and institutional affiliations.

Open Access This article is licensed under a Creative Commons Attribution 4.0 International License, which permits use, sharing, adaptation, distribution and reproduction in any medium or format, as long as you give appropriate credit to the original author(s) and the source, provide a link to the Creative Commons licence, and indicate if changes were made. The images or other third party material in this article are included in the article's Creative Commons licence, unless indicated otherwise in a credit line to the material. If material is not included in the article's Creative Commons licence and your intended use is not permitted by statutory regulation or exceeds the permitted use, you will need to obtain permission directly from the copyright holder. To view a copy of this licence, visit http://creativecommons.org/licenses/by/ $4.0 \%$. 